\title{
Associations between urinary hydration markers and metabolic dysfunction: a cross-sectional analysis of NHANES data, 2008-2010
}

\author{
Tiphaine Vanhaecke ${ }^{1}$ (D) Alberto Dolci ${ }^{1} \cdot$ Victor L. Fulgoni IIII $\cdot$ Harris R. Lieberman ${ }^{3}$
}

Received: 16 November 2020 / Accepted: 27 April 2021 / Published online: 18 May 2021

(c) The Author(s) 2021

\begin{abstract}
Purpose Growing evidence suggests hydration plays a role in metabolic dysfunction, however data in humans are scarce. This study examined the cross-sectional association between hydration and metabolic dysfunction in a representative sample of the US population.

Methods Data from 3961 adult NHANES (National Health and Nutrition Examination Survey) participants (49.8\% female; age $46.3 \pm 0.5$ years) were grouped by quartile of urine specific gravity ( $U_{\mathrm{SG}}, 2007-2008$ cohort) or urine osmolality $\left(U_{\mathrm{Osm}}\right.$, 2009-2010 cohort) as measures of hydration. Metabolic dysfunction was assessed by glycemic and insulinemic endpoints and by components of the metabolic syndrome. Multivariate-adjusted linear and logistic regression models were used.

Results Increasing quartiles of $U_{\mathrm{SG}}$ but not $U_{\mathrm{Osm}}$ was associated with higher fasting plasma glucose (FPG), glycated hemoglobin (all $P<0.01$ ), HOMA-IR and elevated insulin (all $P<0.05$ ). Compared with the lowest quartile, those with the highest $U_{\mathrm{SG}}$ but not $U_{\mathrm{Osm}}$ had greater risk of metabolic syndrome (Q4 vs. Q1, OR $\left.(99 \% \mathrm{CI}): 1.6(1.0,2.7), P=0.01\right)$ and diabetes $(\mathrm{Q} 4$ vs. Q1, OR: $1.8(1.0,3.4), P<0.05)$. Additionally, those with $U_{\mathrm{SG}}>1.013$ or $U_{\mathrm{Osm}}>500 \mathrm{mOsm} / \mathrm{kg}$, common cut-off values for optimal hydration based on retrospective analyses of existing data, had less favorable metabolic markers. In a subset of participants free from diabetes mellitus, impaired kidney function, hypertension and diuretic medication, $U_{\mathrm{SG}}$ remained positively associated with FPG $(P<0.01)$ and elevated FPG $(P<0.05)$.

Conclusion These analyses provide population-based evidence that $U_{\mathrm{SG}}$ as a proxy for hydration is associated with glucose homeostasis in NHANES 2007-2008. The same association was not significant when $U_{\text {Osm }}$ was used as a proxy for hydration in the 2009-2010 wave.
\end{abstract}

Clinical trial registry Not applicable, as this was a reanalysis of existing NHANES data.

Keywords Diabetes $\cdot$ Metabolic syndrome $\cdot$ Hydration $\cdot$ Urine specific gravity $\cdot$ Urine osmolality $\cdot$ NHANES

\section{Abbreviations}

AVP Arginine vasopressin

CDC Centers for Disease Control and Prevention

FPG Fasting plasma glucose

HbA1c Glycated haemoglobin

LS Least square

Tiphaine Vanhaecke and Alberto Dolci contributed equally to this study

Tiphaine Vanhaecke

tiphaine.vanhaecke@danone.com

1 Health, Hydration and Nutrition Science Department, Danone Research, Route Départementale 128, 91767 Palaiseau, France

2 Nutrition Impact, LLC, Battle Creek, MI, USA

3 Newton, MA, USA
NHANES National Health and Nutrition Examination Survey

NCHS National Center for Health Statistics

$U_{\text {Osm }} \quad$ Urine osmolality

$U_{\mathrm{SG}} \quad$ Urine specific gravity

V1aR Vasopressin 1A receptor

WHO World Health Organisation

\section{Introduction}

High fasting plasma glucose is the key element for the development and diagnosis of diabetes, which is currently among the greatest global public health challenges. The World Health Organization estimates that the rate of increase of individuals with high fasting plasma glucose has surpassed that for obesity, and has designated it as the third highest 
factor in mortality rate rankings [1]. Metabolic syndrome, a cluster of glucose intolerance, insulin resistance, hypertension and dyslipidemia is also on the rise, thereby magnifying the risk of diabetes incidence [2,3]. Diet and lifestyle have a direct impact on metabolic function; consequently lifestyle interventions incorporating dietary changes and physical activity may reduce the risk of diabetes in high-risk individuals with impaired glucose regulation $[4,5]$.

Among potential dietary risk factors, water intake and fluid balance processes have rarely been considered. While it is becoming increasingly evident that increased water intake may decrease the risk of metabolic diseases by reducing intake of sugar-sweetened beverage [6,7], the hypothesis that water intake or fluid balance processes may play an independent role in modulating disease risk has not received much attention. Several reports have documented an impairment of glucose metabolism by plasma hypertonicity, an indicator of cellular dehydration $[8,9]$. In this context, dehydration was suggested to be an additional factor contributing to the development of insulin resistance and risk of diabetes. It is only recently that this hypothesis received further attention, with additional evidence suggesting a link between low water intake and the development of metabolic disease. In the short term, acute low water intake was shown to impair glycemic control in men with type-2 diabetes [10]. In the general population, higher water intake has consistently been associated with lower blood glucose levels and risk of diabetes in men, but not in women [11-13]. In particular, in a prospective cohort on French general population, low water intake was associated with new-onset hyperglycemia over a 9-year follow-up period independently of major baseline confounding factors [11]. The authors also reported water intake was inversely associated with urine specific gravity. When used in large population surveys, urinary hydration biomarkers such as urine osmolality $\left(U_{\mathrm{Osm}}\right)$, specific gravity $\left(U_{\mathrm{SG}}\right)$, which are non-invasive measures and objective proxy for water homeostasis, may avoid potential recall bias and inaccuracy associated with self-reported recall of food and beverage intake. Both $U_{\mathrm{Osm}}$ and $U_{\mathrm{SG}}$ vary according to fluid intake volumes and reflect the end-result of all sources of water intake and water loss, as well as dietary solute load, and represent the diuretic and antidiuretic activity of the kidney [14-17]. However, to-date, urinary biomarkers of hydration have rarely been used to evaluate links between hydration and metabolic health outcomes in large epidemiologic studies.

There is evidence that underhydration is common in the general population; e.g. $70 \%$ of the non-acutely ill US population, aged 19-50 years is estimated to have an unmet need for water, as defined by serum sodium outside the normal range as well as a urine osmolality above $500 \mathrm{mOsm} / \mathrm{kg}$ [18]. Therefore, the estimate of the risk of metabolic dysfunction in relation to hydration in a representative sample of the general population provides useful information from a public health perspective. Should the hypothesis of a link between hydration and metabolic health be corroborated by causal research, improving hydration by increasing water intake may provide a simple and inexpensive intervention to help prevent the development of metabolic dysfunction.

The first aim of this study was to determine whether there were associations between urinary markers of hydration and metabolic endpoints in a representative sample of the US population, using data from the National Health and Nutrition Examination Survey (NHANES). A second aim of this study was to test whether individuals above common cut-off values for optimal hydration had less favorable metabolic markers. To account for possible reverse causation, analyses were replicated in a cluster of individuals who did not have some conditions likely affecting urinary biomarkers of hydration.

\section{Methods}

\section{Sample}

NHANES is a series of cross-sectional national surveys conducted in the United States by the Centers for Disease Control and Prevention (CDC) and is designed to assess the health and nutritional status of the population using a stratified, nationally representative sampling design. Detailed survey descriptions, methodology, sampling procedures, laboratory test procedures, and data tables are publicly available (www.cdc.gov.nchs/nhanes/). Ethical committee approval for the collection of NHANES data was obtained from the NCHS Research Ethics Review Board in accordance with the Declaration of Helsinki and participants provided written informed consent. Subsequent analyses of de-identified data are permitted by federal regulations on human subjects research and exempt from further IRB review under $45 \mathrm{CFR}$ 46.101(b)(4).

\section{Data collection and measures}

NHANES includes, among other measures, data from a physical examination and a face-to-face structured interview. Sociodemographic variables collected during the interview included age, sex, ethnicity, total income, current smoking status. The poverty income ratio (ratio of household income to the poverty threshold) was used as the indicator of socioeconomic status in the present analysis. Physical activity was categorized in three levels based on self-reported responses of days of vigorous activity (sedentary, 0-3 days per week; moderate 4-6 days per week; and vigorous, 7 days per week). Anthropometric data (including height, weight, and waist circumference) were measured during the physical 
examination at the mobile examination center (MEC). Urine and blood samples were also collected and processed at the MEC. Sampling and laboratory measurement methods are accessible online (www.cdc.gov.nchs/nhanes/). Urinespecific gravity (2007-2008 cohort) was determined by refractometry (ATAGO PAL-10S, Atago USA, Inc., Bellevue, WA, USA). Urine osmolality (2009-2010 cohort) was measured by freezing point depression osmometer (Osmette II, Precision Systems Inc., Natick, MA, USA). The fasting status of the participants scheduled for morning visits was verified, and laboratory analyses included fasting glucose and insulin, glycated hemoglobin (HbA1c) as well as triglycerides, LDL and HDL cholesterol. Fasting glucose was determined by enzymatic method (hexokinase enzymatic assay). HbA1c was measured by high pressure liquid chromatography (A1c G7 HPLC Glycohemoglobin Analyzer, Tosoh Medics, CA, USA). The analytical method for serum insulin (sandwich ELISA assay, Mercodia, Sweden) was changed part-way through the 2009-2010 cohort (chemiluminescent immunoassay, Elecsys 2010 analyzer, Roche, Switzerland), with a difference in measured insulin values detected between the two methodologies. Using a fractional polynomial regression, the 2010 insulin participant results were increased so they were equivalent to the 2009 insulin results (www.cdc.gov.nchs/nhanes/). HOMA-IR was calculated as glucose*insulin/405 [19]. Triglycerides and HDLcholesterol were determined by two-reagent enzymatic essay (Modular P chemistry analyzer, Roche, Switzerland). LDLcholesterol was estimated using the Friedewald equation (Total cholesterol-HDL cholesterol-Triglyceride/5) [20]. Self-reported data on medical conditions and medications were also collected.

\section{Variable specifications}

BMI categories were defined as underweight $(\mathrm{BMI}<18.5)$, normal weight $(18.5 \leq \mathrm{BMI}<25)$, overweight $(25 \leq \mathrm{BMI}<30)$ and obese $(\mathrm{BMI} \leq 30)[21]$. Elevated waist circumference was defined as $>102 \mathrm{~cm}$ (male) and $>88 \mathrm{~cm}$ (female) [22]; elevated fasting plasma glucose (FPG) $\geq 100$ $\mathrm{mg} / \mathrm{dL}$ (prediabetes) [23] or antidiabetic medication; elevated $\mathrm{HbA} 1 \mathrm{c} \geq 6.5 \%$ (diabetes) [23]; elevated insulin $\geq 15$ $\mu \mathrm{U} / \mathrm{L}[24]$ or antidiabetic medication; elevated HOMAIR $\geq 4.0$ [25] or antidiabetic medication; elevated triglycerides $\geq 150 \mathrm{mg} / \mathrm{dL}$ [26] or antihyperlipidemic medication; reduced $\mathrm{HDL}<40 \mathrm{mg} / \mathrm{dL}$ (male) and $<50 \mathrm{mg} / \mathrm{dL}$ (female) [27] or antihyperlipidemic medication. Hypertension or elevated blood pressure was defined as systolic $\geq 130 \mathrm{mmHg}$ or diastolic $\geq 80 \mathrm{mmHg}$ [28] or hypertension medication. Participants who reported ever being told that they had diabetes or taking antidiabetic medications were classified as having diabetes. Impaired kidney function was defined as glomerular filtration rate $<60 \mathrm{~mL} / \mathrm{min} / 1.73 \mathrm{~m}^{2}$ [29]. Participants who had any three of the following criteria were classified as having metabolic syndrome: elevated waist circumference; elevated fasting plasma glucose; elevated triglycerides; reduced HDL; elevated blood pressure) [30].

\section{Inclusion/exclusion criteria}

The NHANES 2007-2008 and 2009-2010 fasting subsample data (morning visit at the mobile examination center) of individuals aged $\geq 19$ years were used in this study (2007-2008 cohort, $n=2424 ; 2009-2010$ cohort $n=2696$ ). These were the only NHANES cycles either containing $U_{\mathrm{SG}}$ or $U_{\mathrm{Osm}}$ at the time of analyses. After excluding pregnant or lactating females (2007-2008 cohort, $n=39$; 2009-2010 cohort $n=41$ ), as well as individuals missing data for the variables used in this analysis (2007-2008 cohort, $n=547$; 2009-2010 cohort $n=532$ ), the final analysis datasets included $n=1838$ subjects for the 2007-2008 cohort and $n=2123$ subjects for the 2009-2010 cohort (Fig. 1). Sample characteristics are shown in Table 1. Additionally, in a sensitivity analysis, individuals from this subsample that had either diabetes, hypertension, impaired kidney function or were taking diuretic medication were further excluded and analyses were repeated on a subgroup of $n=852$ (2007-2008 cohort) and $n=1024$ (2009-2010 cohort) otherwise healthy adults.

\section{Statistical analysis}

Statistical analyses were performed using SAS 9.4 (Cary, NC, USA). The NHANES fasting subsample survey weights, strata and primary sampling units were used in all analyses using SURVEYMEANS or SURVEYREG procedures of SAS. Respondents were classified by quartiles of $U_{\mathrm{SG}}\left(2007-2008\right.$ cohort) or $U_{\text {Osm }}(2009-2010$ cohort), respectively. Because the hydration measurement method for urine concentration was different for the 2007-2008 and 2009-2010 cohorts, results for each cohort are presented separately. Physiologic outcome variables are treated both as continuous variables and presented as adjusted means by quartile of urine concentration, and as incidence measures presented as percentages. We used linear and logistic regression to test whether increasing quartiles of urinary biomarkers of hydration were associated with metabolic endpoints. Regression models included age, sex, ethnicity, poverty income ratio, physical activity level, current smoking status and Body Mass Index (BMI) as covariates to investigate associations independent of these common confounding factors. For example, BMI is a well-described confounder for metabolic variables and is also known to be associated with hydration; people with higher BMI have higher odds of being inadequately 


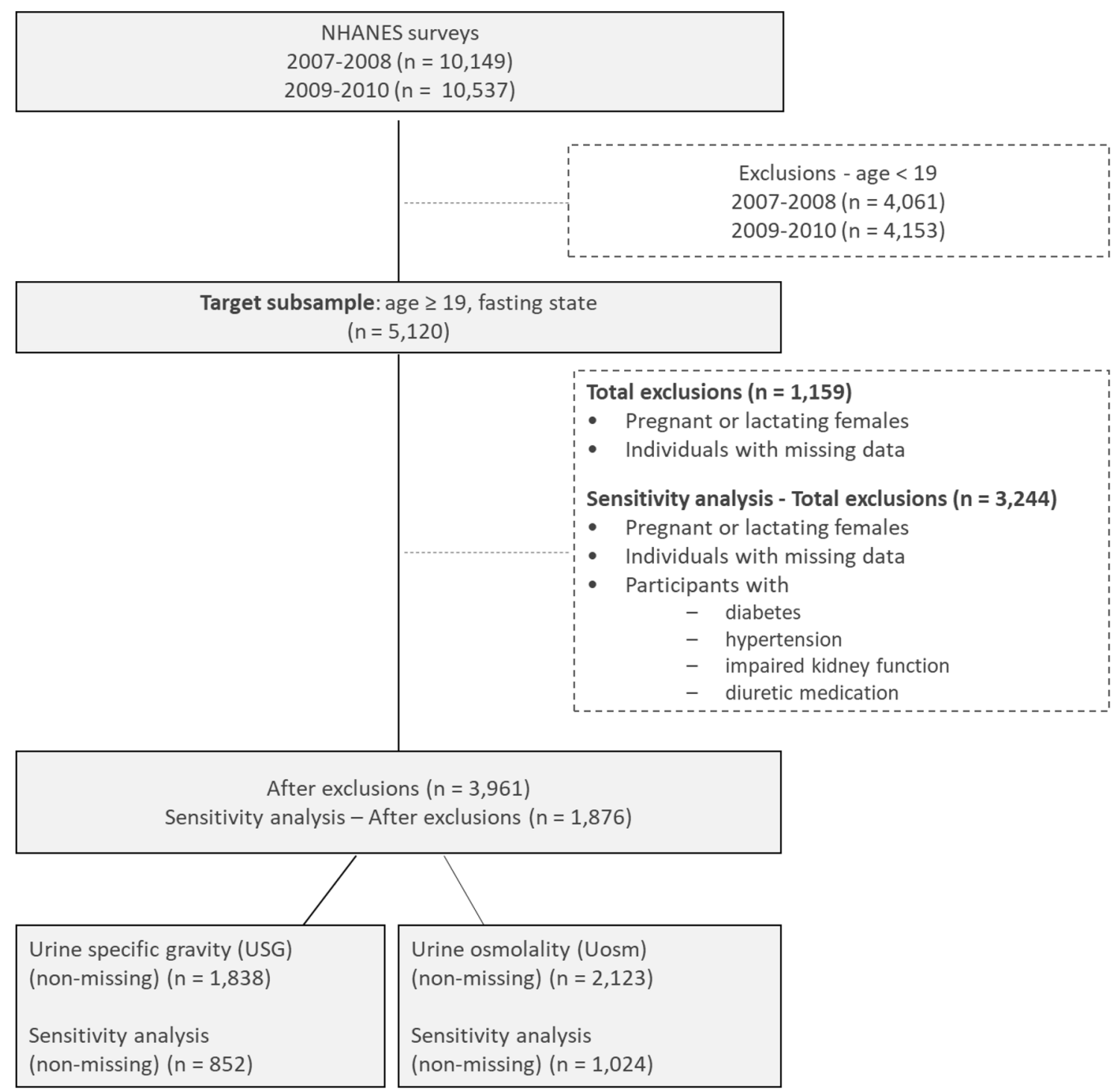

Fig. 1 Participant flowchart from the population of adult participants in the NHANES 2007-2008 and 2009-2010

hydrated $\left(U_{\text {Osm }} \geq 800 \mathrm{mOsm} / \mathrm{kg}\right)[31,32]$. Adjusted least square (LS) means, standard errors (SE) and $P$ values for quartile trend are presented. Data from logistic regression analyses are expressed as odds ratios (ORs) and 95\% confidence intervals (CIs). To test whether individuals above common cut-off values for optimal hydration had less favorable metabolic markers, we explored outcome variables on both sides of a $U_{\text {Osm }}$ cut-off of $500 \mathrm{mOsm} / \mathrm{kg}$, or a $U_{\mathrm{SG}}$ of 1.013 , since these thresholds have previously been proposed as hydration targets for the general population $[33,34]$. Adjusted LS means, SE using regression models with covariates mentioned above are presented and $t$-test were used to assess differences based on cut-offs used.

\section{Results}

\section{Study population}

The present study sample consisted of 1838 individuals in the 2007-2008 cohort and 2123 individuals in the 2009-2010 cohort who had complete information on covariates (Table 1). There were about $50 \%$ females both in the 2007-2008 cohort and 2009-2010 cohort. For descriptive purposes, on average, $40-42 \%$ of the study population had metabolic syndrome and about $10-11 \%$ had self-reported diabetes prior to biological measures at the MEC. 
Table 1 Demographic, physiological and anthropometric characteristics of the study population

\begin{tabular}{|c|c|c|c|c|}
\hline & \multicolumn{2}{|c|}{$2007-2008$} & \multicolumn{2}{|c|}{ 2009-2010 } \\
\hline & Mean & SE & Mean & SE \\
\hline Sample $N$ & 1838 & & 2123 & \\
\hline Age & 45.9 & 0.7 & 46.9 & 0.7 \\
\hline Gender $=$ male $(\%)$ & 49.8 & 1.3 & 50.5 & 1.0 \\
\hline Gender $=$ female $(\%)$ & 50.2 & 1.3 & 49.5 & 1.0 \\
\hline Ethnicity $=$ Mexican American (\%) & 7.9 & 1.5 & 8.0 & 2.0 \\
\hline Ethnicity = Other Hispanic (\%) & 4.1 & 0.9 & 4.3 & 1.1 \\
\hline Ethnicity = Non-Hispanic White & 70.7 & 3.5 & 71.3 & 3.0 \\
\hline Ethnicity $=$ Non-Hispanic Black & 11.4 & 2.3 & 10.1 & 1.0 \\
\hline Body mass index $\left(\mathrm{kg} / \mathrm{m}^{2}\right)$ & 28.3 & 0.2 & 28.6 & 0.2 \\
\hline Underweight $(\mathrm{BMI}<18.5)(\%)$ & 1.7 & 0.4 & 1.6 & 0.4 \\
\hline Normal weight $(18.5 \leq \mathrm{BMI}<25)(\%)$ & 31.0 & 1.2 & 29.8 & 1.8 \\
\hline Overweight $(25 \leq \mathrm{BMI}<30)(\%)$ & 34.9 & 1.5 & 33.2 & 1.3 \\
\hline Obese (BMI $\geq 30)(\%)$ & 32.5 & 1.6 & 35.5 & 1.6 \\
\hline Smoking $=$ current $(\%)$ & 20.3 & 2.2 & 16.9 & 1.4 \\
\hline Hypertension (\%) & 39.4 & 1.4 & 40.4 & 2.2 \\
\hline Glucose (mg/dL) & 105.5 & 0.7 & 103.2 & 0.9 \\
\hline Insulin $(\mu \mathrm{U} / \mathrm{mL})$ & 11.7 & 0.4 & 13.5 & 0.2 \\
\hline HOMA-IR (glucose*insulin/405) & 3.2 & 0.1 & 3.6 & 0.1 \\
\hline HbA1c $(\%)$ & 5.6 & 0.03 & 5.6 & 0.03 \\
\hline Triglyceride (mg/dL) & 123.0 & 2.2 & 118.8 & 2.3 \\
\hline HDL-cholesterol (mg/dL) & 53.3 & 0.7 & 54.2 & 0.6 \\
\hline LDL-cholesterol (mg/dL) & 115.6 & 1.1 & 116.6 & 1.3 \\
\hline Diabetes (\%) & 10.4 & 0.8 & 10.7 & 0.7 \\
\hline Metabolic syndrome (\%) & 41.9 & 1.5 & 40.4 & 1.7 \\
\hline Urine-specific gravity & 1.017 & 0.0002 & & \\
\hline Urine osmolality (mOsm/kg) & & & 610 & 9 \\
\hline
\end{tabular}

Results are sample weighted means or percentages (\%) and standard errors (SE) to ensure national representation. Hypertension (systolic $\geq 130$ or diastolic $\geq 80$ or hypertension medication); diabetes (self-reported diabetes told or taking antidiabetic medications); metabolic syndrome (any three of: elevated waist circumference $(>102$ (male), $>88$ (female)); elevated fasting plasma glucose $(\geq 100 \mathrm{mg} / \mathrm{dL}$ or antidiabetic medication); elevated triglycerides $(\geq 150$ or antihyperlipidemic medication); reduced HDL $(<40$ (male), $<50$ (female) or antihyperlipidemic medication); elevated blood pressure (systolic $\geq 130$ or diastolic $\geq 80$ or hypertension medication)

\section{Associations between $U_{\text {SG }}$ (2007-2008 cohort) or $U_{\text {Osm }}$ (2009-2010 cohort) and glycemic endpoints in the general adult population}

In the 2007-2008 cohort, fasting plasma glucose (FPG) increased with increasing $U_{\mathrm{SG}}$ (Table $2, P<0.01$ ), from (mean $\pm \mathrm{SE}$ ) $101.6 \pm 0.5 \mathrm{mg} / \mathrm{dL}$ in the lowest quartile to $112.1 \pm 1.2 \mathrm{mg} / \mathrm{dL}$ in the top quartile. HbA1c (\%) also increased with increasing $U_{\mathrm{SG}}(P<0.01)$. This trend was not present in the 2009-2010 cohort, with FPG and HbA1c remaining stable across quartiles of $U_{\text {Osm }}$ (Table 3 ).
Higher $U_{\mathrm{SG}}\left(2007-2008\right.$ cohort) but not higher $U_{\mathrm{Osm}}$ (2009-2010 cohort) was also associated with reaching glycemic diagnostic criteria for impaired fasting glucose (Table 2, $P<0.01)$. Individuals in the highest vs. the lowest quartiles for $U_{\mathrm{SG}}$ were more likely to reach thresholds for elevated FPG $(58.0 \pm 2.1 \%$ vs. $48.9 \pm 2.7 \%, P<0.01)$.

\section{Associations between $U_{\mathrm{SG}}$ or $U_{\mathrm{Osm}}$ and insulinemic endpoints}

In the 2007-2008 cohort, insulin resistance (HOMA-IR), increased with urine concentration (Table $2, P<0.05$ ). No statistical association was found between $U_{\text {Osm }}$ and insulinemic endpoints (Table 3 ).

\section{Associations between $U_{S G}$ or $U_{O s m}$ and components of the metabolic syndrome}

In addition to a higher prevalence of elevated FPG, reduced HDL cholesterol was associated with increasing $U_{\mathrm{SG}}$ (Table 2, $P<0.05$ ). Elevated blood pressure was negatively associated with $U_{\mathrm{Osm}}$ (Table $2, P<0.01$ ) but not with $U_{\mathrm{SG}}$ (Table 2). Finally, elevated triglycerides and waist circumference were not associated with urine concentration assessed either by $U_{\mathrm{SG}}$ or $U_{\mathrm{Osm}}\left(U_{\mathrm{SG}}\right.$ : Table $2, U_{\mathrm{Osm}}$ : Table 3$)$.

\section{Associations of $U_{S G}$ and $U_{0 s m}$ with glycemic and insulinemic endpoints and metabolic syndrome in the adult population deemed healthy}

In the segment of the population free from diabetes mellitus, impaired kidney function, hypertension and diuretic medication, conditions which are known to affect hydration status, $U_{\mathrm{SG}}$ but not $U_{\mathrm{Osm}}$ was positively associated with FPG and elevated FPG $\left(P<0.01\right.$ and $P<0.05$, respectively) $\left(U_{\mathrm{SG}}\right.$ : Table 4; $U_{\text {Osm }}$ : Table 5).

\section{Odds ratios for metabolic disease}

Higher $U_{\mathrm{SG}}$ was associated with increased odds of diabetes (Q4 vs. Q1, OR (99\% CI): $1.8(1.0,3.4), P=0.02)$ and metabolic syndrome (Q4 vs. Q1, OR: $1.6(1.0,2.7), P=0.01)$ (Fig. 2, Table S1 (URL: https://figshare.com/s/9521e8960e 6e30550bdc; https://doi.org/10.6084/m9.figshare. 12783 413 ) in the NHANES 2007-2008 cohort. In the segment of the population free from diabetes mellitus, impaired kidney function, hypertension and diuretic medication, higher $U_{\mathrm{SG}}$ remained associated with increased odds of metabolic syndrome (Q4 vs. Q1, OR: $2.7(1.1,6.6), P<0.01)$ (Table S1). There was no association between $U_{\mathrm{Osm}}$ and odds of diabetes or metabolic syndrome in the NHANES 2009-2010 cohort. 
Table 2 Glycemic, insulinemic markers, and metabolic dysfunction endpoints of the adult population of the NHANES 2007-2008 $(n=1838)$ cohort across quartiles of $U_{\mathrm{SG}}$

\begin{tabular}{|c|c|c|c|c|c|}
\hline \multirow[t]{2}{*}{ Quartiles } & Q1 & Q2 & Q3 & Q4 & $P$ for trend \\
\hline & $U_{\mathrm{SG}}<1.011$ & $1.011 \leq U_{\mathrm{SG}}<1.016$ & $1.016 \leq U_{\mathrm{SG}}<1.021$ & \multicolumn{2}{|l|}{$U_{\mathrm{SG}} \geq 1.021$} \\
\hline \multicolumn{6}{|l|}{ Glycemic markers } \\
\hline FPG (mg/dL) & $101.6 \pm 0.5$ & $103.5 \pm 0.7$ & $103.9 \pm 0.8$ & $112.1 \pm 1.2$ & $<0.01$ \\
\hline Elevated FPG & $48.9 \pm 2.7 \%$ & $50.8 \pm 2.8 \%$ & $51.8 \pm 2.5 \%$ & $58.0 \pm 2.0 \%$ & $<0.01$ \\
\hline HbA1c (\%) & $5.50 \pm 0.03$ & $5.54 \pm 0.03$ & $5.51 \pm 0.04$ & $5.67 \pm 0.05$ & $<0.01$ \\
\hline Elevated $\mathrm{HbA} 1 \mathrm{c}$ & $5.5 \pm 1.3 \%$ & $7.4 \pm 1.0 \%$ & $5.8 \pm 1.6 \%$ & $8.9 \pm 1.2 \%$ & 0.09 \\
\hline \multicolumn{6}{|l|}{ Insulinemic markers } \\
\hline Insulin $(\mu \mathrm{U} / \mathrm{L})$ & $11.1 \pm 0.5$ & $11.7 \pm 0.6$ & $11.4 \pm 0.5$ & $12.4 \pm 0.5$ & 0.12 \\
\hline Elevated insulin & $24.1 \pm 2.6 \%$ & $27.6 \pm 2.5 \%$ & $28.0 \pm 2.6 \%$ & $31.7 \pm 2.3 \%$ & 0.04 \\
\hline HOMA-IR & $2.9 \pm 0.1$ & $3.1 \pm 0.2$ & $3.1 \pm 0.2$ & $3.6 \pm 0.2$ & 0.02 \\
\hline Elevated HOMA-IR & $23.1 \pm 2.6 \%$ & $27.0 \pm 2.6 \%$ & $25.5 \pm 2.3 \%$ & $31.1 \pm 1.9 \%$ & 0.04 \\
\hline \multicolumn{6}{|c|}{ Other components of the metabolic syndrome } \\
\hline Elevated waist circumference & $53.0 \pm 2.3 \%$ & $48.9 \pm 1.7 \%$ & $53.0 \pm 2.2 \%$ & $52.8 \pm 1.4 \%$ & 0.70 \\
\hline Elevated triglycerides & $33.8 \pm 2.6 \%$ & $39.6 \pm 1.9 \%$ & $38.9 \pm 2.9 \%$ & $40.3 \pm 1.5 \%$ & 0.04 \\
\hline Reduced HDL cholesterol & $33.1 \pm 2.5 \%$ & $44.2 \pm 2.8 \%$ & $41.4 \pm 2.9 \%$ & $41.9 \pm 1.9 \%$ & 0.02 \\
\hline Elevated BP & $43.2 \pm 3.0 \%$ & $38.3 \pm 2.2 \%$ & $38.6 \pm 2.6 \%$ & $37.7 \pm 2.3 \%$ & 0.27 \\
\hline
\end{tabular}

LS means \pm standard errors and $P$ value for quartile trend are presented. Models were adjusted for age, sex, BMI, ethnicity, poverty income ratio, physical activity level and current smoking status using SURVEYMEANS procedure of SAS. Elevated fasting plasma glucose (FPG) $(\geq 100 \mathrm{mg} / \mathrm{dL}$ or antidiabetic medication); elevated glycated hemoglobin (HbA1c) $(\geq 6.5 \%)$; elevated insulin $(\geq 15 \mu \mathrm{U} / \mathrm{L}$ or antidiabetic medication); HOMA-IR (glucose*insulin/405); elevated HOMA-IR ( $\geq 4.0$ or antidiabetic medication); elevated waist circumference ( $>102$ (male), $>88$ (female)); elevated triglycerides ( $\geq 150$ or antihyperlipidemic medication); reduced HDL $(<40$ (male), $<50$ (female) or antihyperlipidemic medication); elevated BP (systolic $\geq 130$ or diastolic $\geq 80$ or hypertension medication)

Table 3 Glycemic, insulinemic markers, and metabolic dysfunction endpoints of the adult population of the NHANES 2009-2010 $(n=2123)$ cohort across quartiles of $U_{\text {Osm }}$

\begin{tabular}{|c|c|c|c|c|c|}
\hline Quartiles & $\begin{array}{l}\text { Q1 } \\
U_{\mathrm{Osm}}<405\end{array}$ & $\begin{array}{l}\text { Q2 } \\
405 \leq U_{\mathrm{Osm}}<617\end{array}$ & $\begin{array}{l}\text { Q3 } \\
617 \leq U_{\text {Osm }}<808\end{array}$ & $\begin{array}{l}\mathrm{Q} 4 \\
U_{\mathrm{Osm}} \geq 808\end{array}$ & $P$ for trend \\
\hline \multicolumn{6}{|l|}{ Glycemic markers } \\
\hline FPG (mg/dL) & $102.5 \pm 1.1$ & $103.8 \pm 1.1$ & $104.3 \pm 1.8$ & $102.0 \pm 1.1$ & 0.81 \\
\hline Elevated FPG & $46.8 \pm 2.1 \%$ & $49.7 \pm 2.7 \%$ & $45.3 \pm 2.1 \%$ & $44.5 \pm 3.3 \%$ & 0.37 \\
\hline HbA1c $(\%)$ & $5.60 \pm 0.04$ & $5.62 \pm 0.02$ & $5.63 \pm 0.06$ & $5.55 \pm 0.03$ & 0.27 \\
\hline Elevated $\mathrm{HbA} 1 \mathrm{c}$ & $6.7 \pm 1.0 \%$ & $9.4 \pm 1.2 \%$ & $7.4 \pm 1.9 \%$ & $6.0 \pm 1.0 \%$ & 0.32 \\
\hline \multicolumn{6}{|l|}{ Insulinemic markers } \\
\hline Insulin $(\mu \mathrm{U} / \mathrm{L})$ & $13.2 \pm 0.2$ & $13.3 \pm 0.6$ & $13.5 \pm 0.7$ & $13.8 \pm 0.4$ & 0.35 \\
\hline Elevated insulin & $34.3 \pm 2.3 \%$ & $33.7 \pm 3.6 \%$ & $33.9 \pm 2.4 \%$ & $35.2 \pm 2.0 \%$ & 0.79 \\
\hline HOMA-IR & $3.5 \pm 0.1$ & $3.6 \pm 0.2$ & $3.6 \pm 0.2$ & $3.6 \pm 0.1$ & 0.62 \\
\hline Elevated HOMA-IR & $33.4 \pm 1.9 \%$ & $32.9 \pm 3.2 \%$ & $30.8 \pm 2.4 \%$ & $33.0 \pm 1.8 \%$ & 0.71 \\
\hline \multicolumn{6}{|c|}{ Other components of the metabolic syndrome } \\
\hline Elevated waist circumference & $53.3 \pm 2.1 \%$ & $53.7 \pm 1.9 \%$ & $54.5 \pm 1.7 \%$ & $55.1 \pm 2.0 \%$ & 0.43 \\
\hline Elevated triglycerides & $35.1 \pm 1.8 \%$ & $36.8 \pm 2.2 \%$ & $35.6 \pm 2.3 \%$ & $34.9 \pm 4.2 \%$ & 0.92 \\
\hline Reduced HDL cholesterol & $43.0 \pm 2.3 \%$ & $46.2 \pm 2.2 \%$ & $38.9 \pm 2.1 \%$ & $39.7 \pm 3.8 \%$ & 0.20 \\
\hline Elevated BP & $44.7 \pm 2.1 \%$ & $42.0 \pm 2.4 \%$ & $40.8 \pm 3.0 \%$ & $34.4 \pm 2.7 \%$ & $<0.01$ \\
\hline
\end{tabular}

LS means \pm standard errors and $P$ value for quartile trend are presented. Models were adjusted for age, sex, BMI, ethnicity, poverty income ratio, physical activity level and current smoking status using SURVEYMEANS procedure of SAS. Elevated fasting plasma glucose (FPG) ( $\geq 100$ $\mathrm{mg} / \mathrm{dL}$ or antidiabetic medication); elevated glycated hemoglobin (HbA1c) ( $\geq 6.5 \%)$; elevated insulin ( $\geq 15 \mu \mathrm{U} / \mathrm{L}$ or antidiabetic medication); HOMA-IR (glucose*insulin/405); elevated waist circumference ( $>102$ (male), $>88$ (female)); elevated HOMA-IR ( $\geq 4.0$ or antidiabetic medication); elevated triglycerides ( $\geq 150$ or antihyperlipidemic medication); reduced HDL $(<40$ (male), $<50$ (female) or antihyperlipidemic medication); elevated BP (systolic $\geq 130$ or diastolic $\geq 80$ or hypertension medication) 
Table 4 Glycemic, insulinemic markers, and metabolic dysfunction endpoints of the adult population of the NHANES 2007-2008 cohort free from diabetes mellitus, impaired kidney function, hypertension and diuretic medication $(n=852)$ across quartiles of $U_{\mathrm{SG}}$

\begin{tabular}{|c|c|c|c|c|c|}
\hline Quartiles & $\begin{array}{l}\mathrm{Q} 1 \\
U_{\mathrm{SG}}<1.011\end{array}$ & $\begin{array}{l}\mathrm{Q} 2 \\
1.011 \leq U_{\mathrm{SG}}<1.016\end{array}$ & $\begin{array}{l}\mathrm{Q} 3 \\
1.016 \leq U_{\mathrm{SG}}<1.021\end{array}$ & $\begin{array}{l}\mathrm{Q} 4 \\
U_{\mathrm{SG}} \geq 1.021\end{array}$ & $P$ for trend \\
\hline \multicolumn{6}{|l|}{ Glycemic markers } \\
\hline FPG (mg/dL) & $96.3 \pm 0.6$ & $96.8 \pm 0.8$ & $97.1 \pm 0.8$ & $99.0 \pm 0.6$ & $<0.01$ \\
\hline Elevated FPG & $33.5 \pm 4.1 \%$ & $32.9 \pm 3.5 \%$ & $37.0 \pm 4.0 \%$ & $42.2 \pm 2.9 \%$ & 0.03 \\
\hline HbA1c $(\%)$ & $5.32 \pm 0.02$ & $5.29 \pm 0.03$ & $5.29 \pm 0.03$ & $5.26 \pm 0.03$ & 0.13 \\
\hline Elevated $\mathrm{HbA} 1 \mathrm{c}$ & $0.8 \pm 0.6 \%$ & $0.1 \pm 0.1 \%$ & $0.1 \pm 0.1 \%$ & $0.8 \pm 0.4 \%$ & 0.97 \\
\hline \multicolumn{6}{|l|}{ Insulinemic markers } \\
\hline Insulin $(\mu \mathrm{U} / \mathrm{L})$ & $10.0 \pm 0.5$ & $9.5 \pm 0.6$ & $10.1 \pm 0.4$ & $10.6 \pm 0.6$ & 0.32 \\
\hline Elevated insulin & $16.4 \pm 3.1 \%$ & $13.2 \pm 2.6 \%$ & $19.8 \pm 2.9 \%$ & $17.3 \pm 3.1 \%$ & 0.53 \\
\hline HOMA-IR & $2.4 \pm 0.1$ & $2.3 \pm 0.2$ & $2.5 \pm 0.1$ & $2.7 \pm 0.2$ & 0.24 \\
\hline Elevated HOMA-IR & $14.5 \pm 2.9 \%$ & $12.5 \pm 2.7 \%$ & $17.5 \pm 2.0 \%$ & $16.4 \pm 3.0 \%$ & 0.46 \\
\hline \multicolumn{6}{|c|}{ Other components of the metabolic syndrome } \\
\hline Elevated waist circumference & $39.4 \pm 3.1 \%$ & $35.7 \pm 2.6 \%$ & $41.8 \pm 4.0 \%$ & $42.0 \pm 1.9 \%$ & 0.27 \\
\hline Elevated triglycerides & $20.5 \pm 3.7 \%$ & $20.8 \pm 2.9 \%$ & $22.2 \pm 2.8 \%$ & $27.8 \pm 2.9 \%$ & 0.14 \\
\hline Reduced HDL cholesterol & $25.4 \pm 3.9 \%$ & $34.9 \pm 4.6 \%$ & $32.9 \pm 4.2 \%$ & $33.2 \pm 3.9 \%$ & 0.19 \\
\hline
\end{tabular}

LS means \pm standard errors and $P$ value for quartile trend are presented. Models were adjusted for age, sex, BMI, ethnicity, poverty income ratio, physical activity level and current smoking status using SURVEYMEANS procedure of SAS. Elevated fasting plasma glucose (FPG) ( $\geq 100$ $\mathrm{mg} / \mathrm{dL}$ or antidiabetic medication); elevated glycated hemoglobin (HbA1c) $(\geq 6.5 \%)$; elevated insulin ( $\geq 15 \mu \mathrm{U} / \mathrm{L}$ or antidiabetic medication); HOMA-IR (glucose*insulin/405); elevated waist circumference ( $>102$ (male), $>88$ (female)); elevated HOMA-IR ( $\geq 4.0$ or antidiabetic medication); elevated triglycerides ( $\geq 150$ or antihyperlipidemic medication); reduced HDL $(<40$ (male), $<50$ (female) or antihyperlipidemic medication)

Table 5 Glycemic, insulinemic markers, and metabolic dysfunction endpoints of the adult population of the NHANES 2009-2010 cohort free from diabetes mellitus, impaired kidney function, hypertension and diuretic medication $(n=1024)$ across quartiles of $U_{\text {Osm }}$

\begin{tabular}{|c|c|c|c|c|c|}
\hline Quartiles & $\begin{array}{l}\text { Q1 } \\
U_{\text {Osm }}<376 \\
\end{array}$ & $\begin{array}{l}\text { Q2 } \\
376 \leq U_{\mathrm{Osm}}<653\end{array}$ & $\begin{array}{l}\mathrm{Q} 3 \\
653 \leq U_{\mathrm{Osm}}<852\end{array}$ & $\begin{array}{l}\mathrm{Q} 4 \\
U_{\mathrm{Osm}} \geq 852 \\
\end{array}$ & $P$ for trend \\
\hline \multicolumn{6}{|l|}{ Glycemic markers } \\
\hline FPG (mg/dL) & $97.0 \pm 0.8$ & $96.4 \pm 1.0$ & $95.6 \pm 0.9$ & $95.2 \pm 0.7$ & 0.06 \\
\hline Elevated FPG & $32.1 \pm 2.7 \%$ & $35.4 \pm 3.2 \%$ & $28.1 \pm 3.1 \%$ & $28.5 \pm 3.8 \%$ & 0.28 \\
\hline HbA1c (\%) & $5.41 \pm 0.02$ & $5.32 \pm 0.03$ & $5.35 \pm 0.02$ & $5.30 \pm 0.03$ & 0.04 \\
\hline Elevated HbA1c & $0.7 \pm 0.3 \%$ & $1.0 \pm 0.7 \%$ & $0.9 \pm 0.8 \%$ & $0.2 \pm 0.3 \%$ & 0.37 \\
\hline \multicolumn{6}{|l|}{ Insulinemic markers } \\
\hline Insulin $(\mu \mathrm{U} / \mathrm{L})$ & $11.7 \pm 0.7$ & $10.8 \pm 0.5$ & $11.5 \pm 0.5$ & $12.0 \pm 0.7$ & 0.64 \\
\hline Elevated insulin & $20.6 \pm 2.0 \%$ & $16.8 \pm 2.4 \%$ & $23.7 \pm 2.6 \%$ & $24.6 \pm 3.4 \%$ & 0.20 \\
\hline HOMA-IR & $2.9 \pm 0.2$ & $2.6 \pm 0.1$ & $2.8 \pm 0.2$ & $2.9 \pm 0.2$ & 0.78 \\
\hline Elevated HOMA-IR & $20.6 \pm 1.8 \%$ & $15.3 \pm 2.6 \%$ & $16.2 \pm 2.5 \%$ & $20.7 \pm 3.2 \%$ & 0.95 \\
\hline \multicolumn{6}{|c|}{ Other components of the metabolic syndrome } \\
\hline Elevated waist circumference & $37.3 \pm 1.4 \%$ & $43.1 \pm 3.4 \%$ & $41.2 \pm 2.6 \%$ & $39.6 \pm 1.6 \%$ & 0.57 \\
\hline Elevated triglycerides & $19.4 \pm 2.7 \%$ & $24.2 \pm 3.6 \%$ & $29.4 \pm 2.9 \%$ & $22.1 \pm 4.5 \%$ & 0.14 \\
\hline Reduced HDL cholesterol & $33.6 \pm 3.1 \%$ & $36.7 \pm 5.1 \%$ & $29.4 \pm 2.9 \%$ & $25.3 \pm 3.6 \%$ & 0.09 \\
\hline
\end{tabular}

LS means \pm standard errors and $P$ value for quartile trend are presented. Models were adjusted for age, sex, BMI, ethnicity, poverty income ratio, physical activity level and current smoking status using SURVEYMEANS procedure of SAS. Elevated fasting plasma glucose (FPG) ( $\geq 100 \mathrm{mg} / \mathrm{dL}$ or antidiabetic medication); elevated glycated hemoglobin (HbA1c) ( $\geq 6.5 \%)$; elevated insulin $(\geq 15 \mu \mathrm{U} / \mathrm{L}$ or antidiabetic medication); HOMA-IR (glucose*insulin/405); elevated waist circumference ( $>102$ (male), $>88$ (female)); elevated HOMA-IR ( $\geq 4.0$ or antidiabetic medication); elevated triglycerides $(\geq 150$ or antihyperlipidemic medication); reduced HDL $(<40$ (male), $<50$ (female) or antihyperlipidemic medication) 

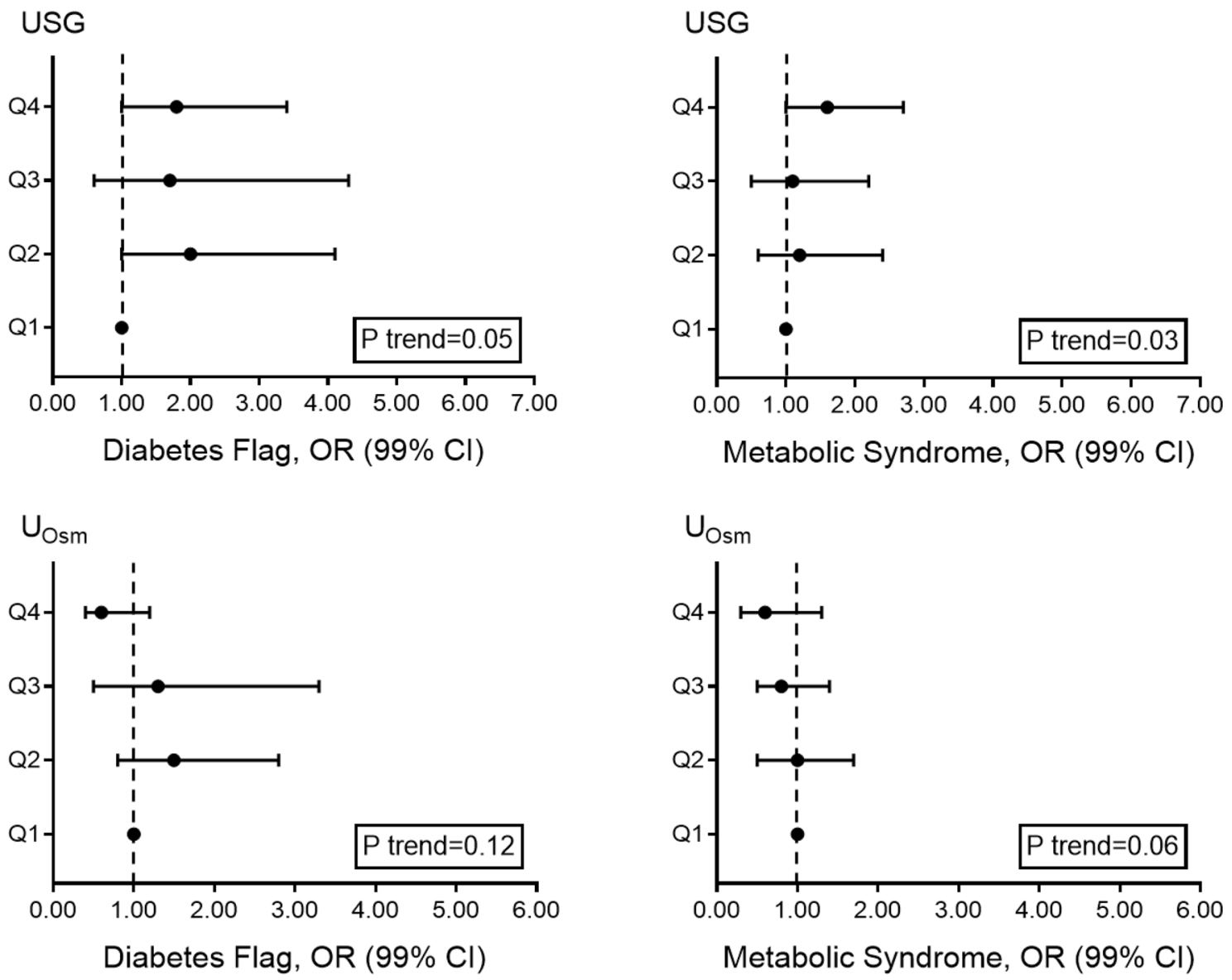

Fig. 2 Adjusted odds ratios (OR) and 99\% confidence interval (CI) for metabolic syndrome and diabetes mellitus in relation to quartiles of $U_{\mathrm{SG}}(N=1838)$ and $U_{\mathrm{Osm}}(N=2123)$. OR were adjusted for age, sex, BMI, ethnicity, poverty income ratio, physical activity level and current smoking status. Diabetes flag (diabetes told or antidiabetic medication); metabolic syndrome (any three of: elevated waist cir-

\section{Differences in metabolic markers between well-hydrated $\left(U_{\text {Osm }} \leq 500 \mathrm{mOsm} / \mathrm{kg}\right.$; $\left.U_{S G} \leq 1.013\right)$ and under-hydrated $\left(U_{O s m}>500 \mathrm{mOsm} /\right.$ kg; $\left.U_{S G}>1.013\right)$ individuals}

Compared to those with $U_{\mathrm{SG}} \leq 1.013$, individuals with higher $U_{\mathrm{SG}}$ had higher glucose (Table $6, P<0.01$ ) whereas individuals with $U_{\mathrm{Osm}}>500 \mathrm{mOsm} / \mathrm{kg}$ had higher insulin $(P<0.01)$ compared to those with lower $U_{\text {Osm }}$. After excluding participants with diabetes mellitus, impaired kidney function, hypertension and diuretic medication, $U_{\mathrm{SG}}$ remained positively associated with glucose $(P<0.01)$.

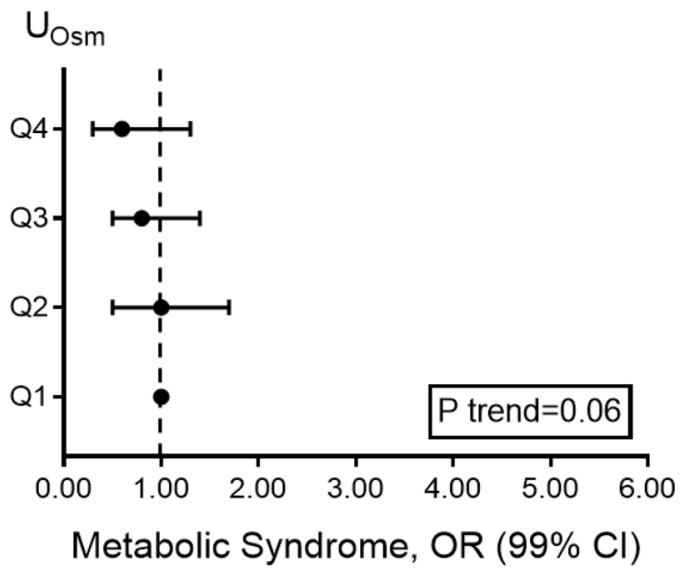

cumference $(>102$ (male), $>88$ (female)); elevated fasting plasma glucose (FPG) ( $\geq 100 \mathrm{mg} / \mathrm{dL}$ or antidiabetic medication); elevated triglycerides ( $\geq 150$ or antihyperlipidemic medication); reduced HDL ( $<40$ (male),$<50$ (female) or antihyperlipidemic medication); elevated blood pressure (BP) (systolic $\geq 130$ or diastolic $\geq 80$ or hypertension medication)); odds ratios (OR); Quartile (Q)

\section{Discussion}

This cross-sectional analysis of a representative sample of the US population revealed significant relationships between hydration and some measures of metabolic health. While several previous investigations have examined relationships between water, fluid intake $[10,11,13]$ or vasopressin (copeptin), a key hormone in the regulation of body fluids [35-37] and metabolic outcomes, urinary biomarkers of hydration have seldom been studied in relation to metabolic outcomes [18, 31, 38]. 
Table 6 Glycemic and insulinemic markers in relation to $U_{\mathrm{SG}}$ and $U_{\mathrm{Osm}}$ thresholds for optimal hydration

\begin{tabular}{|c|c|c|c|c|c|c|}
\hline & \multicolumn{3}{|l|}{$U_{\mathrm{SG}}$} & \multicolumn{3}{|l|}{$U_{\mathrm{Osm}}$} \\
\hline & $\leq 1.013$ & $>1.013$ & $P$ value & $\leq 500 \mathrm{mOsm} / \mathrm{kg}$ & $>500 \mathrm{mOsm} / \mathrm{kg}$ & $P$ value \\
\hline \multicolumn{7}{|l|}{ Glycemic markers } \\
\hline \multicolumn{7}{|l|}{$\mathrm{FPG}(\mathrm{mg} / \mathrm{dL})$} \\
\hline Study population & $101.8 \pm 0.6$ & $107.4 \pm 0.6$ & $<0.01$ & $101.9 \pm 1.0$ & $103.8 \pm 1.0$ & 0.05 \\
\hline Healthy subsample & $95.9 \pm 0.5$ & $98.1 \pm 0.5$ & $<0.01$ & $96.6 \pm 0.6$ & $95.8 \pm 0.7$ & 0.27 \\
\hline \multicolumn{7}{|l|}{ HbA1c (\%) } \\
\hline Study population & $5.51 \pm 0.02$ & $5.58 \pm 0.03$ & 0.06 & $5.58 \pm 0.04$ & $5.61 \pm 0.02$ & 0.48 \\
\hline Healthy subsample & $5.3 \pm 0.02$ & $5.3 \pm 0.02$ & 0.43 & $5.4 \pm 0.02$ & $5.3 \pm 0.01$ & 0.08 \\
\hline \multicolumn{7}{|l|}{ Insulinemic markers } \\
\hline \multicolumn{7}{|l|}{ Insulin $(\mu \mathrm{U} / \mathrm{L})$} \\
\hline Study population & $11.2 \pm 0.4$ & $12.0 \pm 0.4$ & 0.12 & $13.3 \pm 0.4$ & $14.2 \pm 0.3$ & $<0.01$ \\
\hline Healthy subsample & $9.7 \pm 0.4$ & $10.2 \pm 0.4$ & 0.40 & $11.5 \pm 0.5$ & $11.5 \pm 0.4$ & 0.96 \\
\hline \multicolumn{7}{|l|}{ HOMA-IR } \\
\hline Study population & $2.9 \pm 0.1$ & $3.3 \pm 0.1$ & 0.02 & $3.5 \pm 0.2$ & $3.6 \pm 0.1$ & 0.78 \\
\hline Healthy subsample & $2.4 \pm 0.1$ & $2.5 \pm 0.1$ & 0.30 & $2.8 \pm 0.2$ & $2.8 \pm 0.1$ & 0.93 \\
\hline
\end{tabular}

LS means \pm standard errors and $P$ values from $t$-tests are presented. Models are adjusted for age, sex, BMI, ethnicity, poverty income ratio, physical activity level and current smoker status in the study population $\left(U_{\mathrm{SG}}: 2007-2008(n=1838)\right.$; $\left.U_{\mathrm{Osm}}: 2009-2010(n=2123)\right)$ and in a subset of participants free from diabetes mellitus, impaired kidney function, hypertension and diuretic medication $\left(U_{\mathrm{SG}}: 2007-2008(n=852)\right.$; $U_{\text {Osm }}$ : 2009-2010 $(n=1024)$ ); fasting plasma glucose (FPG); glycated hemoglobin (HbA1c); HOMA-IR (glucose*insulin/405)
Specifically, we show that in both the general population as well as in a subset of healthy individuals, more concentrated urine (as measured by specific gravity) was associated with some glycemic and insulinemic markers. Puzzlingly, these associations were not replicated in the subsequent NHANES cohort, where only a few relationships between hydration and metabolic outcomes were found to be significant. These latter results were consistent with a recent analysis of NHANES 2009-2012 which showed that the prevalence of underhydration, defined as serum sodium $>145 \mathrm{mmol} / \mathrm{L}$, spot urine volume $<50 \mathrm{~mL}$, and/or spot urine osmolality $\geq 500 \mathrm{mmol} / \mathrm{kg}$, was not higher among individuals with diabetes, elevated glucose or elevated $\mathrm{HbA1c}$ than among individuals without these conditions [18]. Although $U_{\mathrm{SG}}$ and $U_{\mathrm{Osm}}$ are well correlated under normal physiological conditions [39], the inherent differences that exist between urine osmolality and urine specific gravity may partly explain the discrepancies found between the two cohorts. Urine osmolality is the number of molecules per kilogram of water contained in urine while urine specific gravity is a comparison of the density of urine to that of water [40]. Therefore, specific gravity is affected by the number of molecules and their molecular weights while osmolality is not. In most cases, $U_{\mathrm{Osm}}$ and $U_{\mathrm{SG}}$ are linearly correlated but if many high-molecular-weight molecules are present in the urine, $U_{\mathrm{SG}}$ will overestimate the urine solute concentration, whereas $U_{\mathrm{Osm}}$ remains accurate [40]. Additionally, because $U_{\mathrm{SG}}$ and $U_{\mathrm{Osm}}$ present a different distribution, where the former is linear, and the latter is exponential we could expect that where there are associations with one, there are not necessarily associations with the other. Finally, by accounting for confounding factors and having provided cut-off values, we excluded the values at the 2-ends of the distribution where $U_{\mathrm{Osm}}$ shows most of the differences compared to $U_{\mathrm{SG}}$.

Overall, these findings provide additional populationbased evidence on physiological pathways which link water intake, changes in circulating vasopressin (AVP) and metabolic health [41]. It is well established that the end-result of antidiuretic activity of AVP acting on the kidney is maintenance of water balance in response to varying levels of water intake and loss and is well reflected by urine concentration, measured by osmolality, specific gravity, or color [14-17, $42,43]$. For example, the concentration of AVP has been shown to differ between low- and high-drinkers [16, 44], and it has recently been reported that increasing water intake can reduce plasma AVP/copeptin, both in the short term (over several hours or days) [44, 45], and over several weeks [46].

The evidence that concentrated urine is associated with a worse glycemic profile is consistent with recent findings from another large population-based sample, the Malmö Offspring Study, in which high urine osmolality was associated with unfavorable glucometabolic profile [38]. Additionally, an observational study which primarily aimed to describe the determinants of urine osmolality (medical condition, socio-demographic and lifestyle factors) using the NHANES 2009-2012 cohort reported lower blood glucose in participants with very diluted urine but did not explore this 
association in multivariate adjusted models [31]. However, the study reported no association between the multivariate adjusted odds ratios for very dilute or very concentrated urine and diabetes, a finding in line with our results. Additionally, elevated blood glucose (hyperglycemia, elevated $\mathrm{HbA1c)}$ was recently associated with higher copeptin in recent a cross-sectional study [38], and the reduction in blood glucose following water supplementation was found to be driven by individuals with higher baseline copeptin and greater copeptin reduction in a recent small intervention study [47]. However, while many studies have observed links between metabolic health and evidence of underhydration or challenges to water homeostasis, whether it is measured by water intake, urine concentration or copeptin (AVP), the evidence remains inconsistent. Evidence of associations between water intake and glycemic parameters vary depending on the glycemic status of the population studied, the severity of the glucometabolic disorder and sex. In normoglycemic men or in men free from diabetes, water intake is inversely and independently associated with the risk of developing hyperglycemia [11] and associated with lower likelihood of having elevated HbA1c [13]; whereas in studies that did not exclude subjects based on glycemic parameters, no associations were found between glycemic parameters and water intake [11,38]. Collectively, these findings suggest a potential relationship between water intake and glucometabolic disorders, with a stronger association in men than women.

A body of research also has found positive associations between euhydration or water homeostasis and favorable insulinemic profile [10, 37, 48, 49]. To our knowledge, our study was the first population-based study to specifically explore urine concentration in relation to metabolic endpoints in multivariate adjusted models. In addition to measures of insulinemic and glycemic parameters, evidence of underhydration and challenges to water homeostasis have been associated with several components of metabolic syndrome, including adiposity (higher waist circumference or abdominal obesity) [38, 49-51] and dyslipidemia (lower HDL cholesterol, higher triglycerides) $[38,49,51]$ in multivariate adjusted models. Our current study also found positive associations between a marker of adiposity (lower HDL cholesterol) or the risk of metabolic syndrome and $U_{\mathrm{SG}}$.

Finally, it should also be noted that in individuals with higher copeptin, the risk of developing diabetes may be increased, even after adjusting for a wide range of confounding factors [36, 48-50, 52]. This is observed even in subsets of normoglycemic individuals at baseline [36, 48, 52]. Abbasi et al. reported sex differences in the PREVEND cohort, with the association between plasma copeptin and incident diabetes found in women but not in men [52]. On the contrary, Pan et al. [12] reported no decreased risk of type-2 diabetes with increased water consumption in a large, female-only cohort. While these two findings provide supportive evidence, they are difficult to compare as the former used plasma copeptin and the latter used self-reported water intake as independent variables. This inconsistency suggests that additional studies should be conducted to clarify how water and hydration influence diabetes risk in men and women.

There are areas in which the existing findings are conflicting or inconclusive. Many of the associations between $U_{\mathrm{SG}}$ and metabolic outcomes in the 2007-2008 cohort were not found in the next cohort; for example, fasting plasma glucose and the incidence of diabetes were related to $U_{\mathrm{SG}}$ in 2007-2008, but this finding was not confirmed in the 2009-2010 cohort which used $U_{\mathrm{Osm}}$ as a measure of urine concentration. This explains why results for each cohort are presented separately and is perhaps one of the factors contributing to the lack of consistency in our findings. Furthermore, the analytical method for serum insulin was changed part-way through the 2009-2010 cohort, with a difference in measured insulin values detected between the two methodologies (www.cdc.gov.nchs/nhanes/). While a correction factor was applied to the values obtained in 2010 to bring them in line with the 2009 values, this may be another source of imprecision that may have contributed to the inconsistency in results. We also acknowledge the limitations of a single, morning, spot urine sample as an objective proxy for hydration: morning urine samples are less likely to represent 24-h urine concentration, which is more reflective of fluid intake. This may further explain the discrepancies in the associations observed between hydration and metabolic outcomes between the two cohorts. However, because of the nature of the metabolic outcomes we were interested in studying, only participants having completed fasted-state, morning visits (and thus providing morning urine samples) were included in this analysis. To minimize the impact of this limitation, analyses by quartile of urine concentration were conducted, as it has been shown that low-volume drinkers have significantly higher urine concentration than high-volume drinkers, even in morning samples [16, 17]. Moreover, spot urine samples are the most practical measure of hydration in such large cohorts and provide an opportunity to study a relevant hydration biomarker linked to fluid intake, AVP, and metabolic health outcomes in large representative population samples. Although our analyses have consistently adjusted for a wide range of demographic, socio-economic, lifestyle and biological confounding factors, relevant environmental factors such as the season of the examination, and dietary factors such as salt intake, protein intake or total energy intake were not considered in this analysis and may have affected the results. There were also issues of residual confounders due to events that occurred prior to conduct of the current analysis. Current smoking status was considered, thereby ignoring any change in smoking behavior in previous years. As for any cross-sectional study, there is a risk of reverse causation. However, exclusion of individuals 
with either diabetes, hypertension, impaired kidney function or taking diuretic medication in a sensitivity analysis is likely to have limited the risk of reverse causation. Finally, one of the strengths of this study is that it is an analysis of a nationally representative sample of the population and uses objective urinary biomarkers of hydration that provide an accurate measure of hydration at the population level.

Future research should establish whether a causal link exists between high urine concentration due to low or insufficient fluid intake and metabolic dysfunction. Furthermore, studies should be conducted to determine whether these physiological indicators can be used to define a target fluid intake for optimal hydration that compensates for water losses and maintains urine output that reduces the risk of metabolic dysfunction.

Supplementary Information The online version contains supplementary material available at https://doi.org/10.1007/s00394-021-02575-3.

Acknowledgements The authors are indebted to Dr. Erica T. Perrier (Danone Research) for the advice provided on study design and manuscript writing.

Author contribution All authors designed the research; V. L. F. conducted analyses; all authors analyzed the data; T. V. and A. D. wrote the manuscript and had primary responsibility for final content; all authors read and approved the final manuscript.

Funding This work was supported by Danone Research, Palaiseau, France.

Availability of data and material Detailed NHANES survey descriptions, methodology, sampling procedures, laboratory test procedures, and data tables are publicly available (www.cdc.gov.nchs/nhanes/).

Code availability Not applicable.

\section{Declarations}

Conflict of interest T. V. and A. D. are full-time employees of Danone Research. V. L. F. performs consulting and database analyses for various food and beverage companies and related entities as Senior Vice President of Nutrition Impact, LLC. H. R. L. is a consultant for Danone Research.

Ethics approval/consent to participate/consent for publication Ethical committee approval for the collection of NHANES data was obtained from the NCHS Research Ethics Review Board in accordance with the Declaration of Helsinki and participants provided written informed consent. Subsequent analyses of de-identified data are permitted by federal regulations on human subjects research and exempt from further IRB review under 45 CFR 46.101(b)(4).

Open Access This article is licensed under a Creative Commons Attribution 4.0 International License, which permits use, sharing, adaptation, distribution and reproduction in any medium or format, as long as you give appropriate credit to the original author(s) and the source, provide a link to the Creative Commons licence, and indicate if changes were made. The images or other third party material in this article are included in the article's Creative Commons licence, unless indicated otherwise in a credit line to the material. If material is not included in the article's Creative Commons licence and your intended use is not permitted by statutory regulation or exceeds the permitted use, you will need to obtain permission directly from the copyright holder. To view a copy of this licence, visit http://creativecommons.org/licenses/by/4.0/.

\section{References}

1. World Health Organization (2009) Global health risks: mortality and burden of disease attributable to selected major risks

2. Shin J-A, Lee J-H, Lim S-Y, Ha H-S, Kwon H-S, Park Y-M, Lee W-C, Kang M-I, Yim H-W, Yoon K-H, Son H-Y (2013) Metabolic syndrome as a predictor of type 2 diabetes, and its clinical interpretations and usefulness. J Diabetes Investig 4(4):334-343. https://doi.org/10.1111/jdi.12075

3. Saklayen MG (2018) The global epidemic of the metabolic syndrome. Curr Hypertens Rep 20(2):12-12. https://doi.org/ 10.1007/s11906-018-0812-z

4. Tuomilehto J, Lindstrom J, Eriksson JG, Valle TT, Hamalainen H, Ilanne-Parikka P, Keinanen-Kiukaanniemi S, Laakso M, Louheranta A, Rastas M, Salminen V, Uusitupa M (2001) Prevention of type 2 diabetes mellitus by changes in lifestyle among subjects with impaired glucose tolerance. N Engl J Med 344(18):1343-1350. https://doi.org/10.1056/nejm20010503344 1801

5. Knowler WC, Barrett-Connor E, Fowler SE, Hamman RF, Lachin JM, Walker EA, Nathan DM (2002) Reduction in the incidence of type 2 diabetes with lifestyle intervention or metformin. N Engl J Med 346(6):393-403. https://doi.org/10.1056/NEJMoa012512

6. Tate DF, Turner-McGrievy G, Lyons E, Stevens J, Erickson K, Polzien K, Diamond M, Wang X, Popkin B (2012) Replacing caloric beverages with water or diet beverages for weight loss in adults: main results of the Choose Healthy Options Consciously Everyday (CHOICE) randomized clinical trial. Am J Clin Nutr 95(3):555-563. https://doi.org/10.3945/ajcn.111.026278

7. Romaguera D, Norat T, Wark PA, Vergnaud AC, Schulze MB, van Woudenbergh GJ, Drogan D, Amiano P, Molina-Montes E, Sanchez MJ, Balkau B, Barricarte A, Beulens JW, ClavelChapelon F, Crispim SP, Fagherazzi G, Franks PW, Grote VA, Huybrechts I, Kaaks R, Key TJ, Khaw KT, Nilsson P, Overvad K, Palli D, Panico S, Quiros JR, Rolandsson O, Sacerdote C, Sieri S, Slimani N, Spijkerman AM, Tjonneland A, Tormo MJ, Tumino $\mathrm{R}$, van den Berg SW, Wermeling PR, Zamara-Ros R, Feskens EJ, Langenberg C, Sharp SJ, Forouhi NG, Riboli E, Wareham NJ (2013) Consumption of sweet beverages and type 2 diabetes incidence in European adults: results from EPIC-InterAct. Diabetologia 56(7):1520-1530. https://doi.org/10.1007/s00125-013-2899-8

8. Bratusch-Marrain PR, DeFronzo RA (1983) Impairment of insulin-mediated glucose metabolism by hyperosmolality in man. Diabetes 32(11):1028-1034. https://doi.org/10.2337/diab.32.11. 1028

9. Stookey JD, Pieper CF, Cohen HJ (2004) Hypertonic hyperglycemia progresses to diabetes faster than normotonic hyperglycemia. Eur J Epidemiol 19(10):935-944. https://doi.org/10.1007/ s10654-004-5729-y

10. Johnson EC, Bardis CN, Jansen LT, Adams JD, Kirkland TW, Kavouras SA (2017) Reduced water intake deteriorates glucose regulation in patients with type 2 diabetes. Nutr Res 43:25-32. https://doi.org/10.1016/j.nutres.2017.05.004

11. Roussel R, Fezeu L, Bouby N, Balkau B, Lantieri O, AlhencGelas F, Marre M, Bankir L, Group DESIRS (2011) Low water intake and risk for new-onset hyperglycemia. Diabetes Care 34(12):2551-2554. https://doi.org/10.2337/dc11-0652 
12. Pan A, Malik VS, Schulze MB, Manson JE, Willett WC, Hu FB (2012) Plain-water intake and risk of type 2 diabetes in young and middle-aged women. Am J Clin Nutr 95(6):1454-1460. https:// doi.org/10.3945/ajcn.111.032698

13. Carroll HA, Betts JA, Johnson L (2016) An investigation into the relationship between plain water intake and glycated $\mathrm{Hb}(\mathrm{HbA} 1 \mathrm{c})$ : a sex-stratified, cross-sectional analysis of the UK National Diet and Nutrition Survey (2008-2012). Br J Nutr 116(10):1-11. https://doi.org/10.1017/S0007114516003688

14. Armstrong LE, Pumerantz AC, Fiala KA, Roti MW, Kavouras SA, Casa DJ, Maresh CM (2010) Human hydration indices: acute and longitudinal reference values. Int J Sport Nutr Exerc Metab 20(2):145-153. https://doi.org/10.1123/ijsnem.20.2.145

15. Armstrong LE, Johnson EC, Munoz CX, Swokla B, Le Bellego L, Jimenez L, Casa DJ, Maresh CM (2012) Hydration biomarkers and dietary fluid consumption of women. J Acad Nutr Diet 112(7):1056-1061. https://doi.org/10.1016/j.jand.2012.03.036

16. Perrier E, Vergne S, Klein A, Poupin M, Rondeau P, Le Bellego L, Armstrong LE, Lang F, Stookey J, Tack I (2013) Hydration biomarkers in free-living adults with different levels of habitual fluid consumption. Br J Nutr 109(9):1678-1687. https://doi.org/ 10.1017/S0007114512003601

17. Perrier E, Demazieres A, Girard N, Pross N, Osbild D, Metzger D, Guelinckx I, Klein A (2013) Circadian variation and responsiveness of hydration biomarkers to changes in daily water intake. Eur J Appl Physiol 113(8):2143-2151. https://doi.org/10.1007/ s00421-013-2649-0

18. Stookey JD, Kavouras S, Suh H, Lang F (2020) Underhydration is associated with obesity, chronic diseases, and death within 3 to 6 Years in the U.S. population aged 51-70 years. Nutrients 12(4). https://doi.org/10.3390/nu12040905

19. Matthews DR, Hosker JP, Rudenski AS, Naylor BA, Treacher DF, Turner RC (1985) Homeostasis model assessment: insulin resistance and beta-cell function from fasting plasma glucose and insulin concentrations in man. Diabetologia 28(7):412-419

20. Friedewald WT, Levy RI, Fredrickson DS (1972) Estimation of the concentration of low-density lipoprotein cholesterol in plasma, without use of the preparative ultracentrifuge. Clin Chem 18(6):499-502

21. Centers for Disease Control and Prevention-Overweight and obesity—defining adult overweight and obesity. https://www.cdc. gov/obesity/adult/defining.html. Accessed April 2021

22. Lean ME, Han TS, Morrison CE (1995) Waist circumference as a measure for indicating need for weight management. BMJ 311(6998):158-161. https://doi.org/10.1136/bmj.311.6998.158

23. Centers for Disease Control and Prevention-Diabetes-Diabetes tests. https://www.cdc.gov/diabetes/basics/getting-tested.html

24. Harris MI, Cowie CC, Gu K, Francis ME, Flegal K, Eberhardt MS (2002) Higher fasting insulin but lower fasting C-peptide levels in African Americans in the US population. Diabetes Metab Res Rev 18(2):149-155. https://doi.org/10.1002/dmrr.273

25. Caporaso NE, Jones RR, Stolzenberg-Solomon RZ, Medgyesi DN, Kahle LL, Graubard BI (2020) Insulin resistance in healthy US adults: findings from the national health and nutrition examination survey (NHANES). Cancer Epidemiol Biomarkers Prevent 29(1):157-168. https://doi.org/10.1158/1055-9965.epi-19-0206

26. Fan W, Philip S, Granowitz C, Toth PP, Wong ND (2020) Prevalence of US adults with triglycerides $\geq 150 \mathrm{mg} / \mathrm{dl}$ : NHANES 2007-2014. Cardiol Ther 9(1):207-213. https://doi.org/10.1007/ s40119-020-00170-x

27. Mayo Clinic-What are optimal levels of HDL cholesterol? https://www.mayoclinic.org/diseases-conditions/high-blood-chole sterol/in-depth/hdl-cholesterol/art-20046388

28. Whelton PK, Carey RM, Aronow WS, Casey DE Jr, Collins KJ, Dennison Himmelfarb C, DePalma SM, Gidding S, Jamerson KA, Jones DW, MacLaughlin EJ, Muntner P, Ovbiagele B, Smith
SC Jr, Spencer CC, Stafford RS, Taler SJ, Thomas RJ, Williams KA Sr, Williamson JD, Wright JT Jr (2018) 2017 ACC/AHA/ AAPA/ABC/ACPM/AGS/APhA/ASH/ASPC/NMA/PCNA guideline for the prevention, detection, evaluation, and management of high blood pressure in adults: executive summary: a report of the American College of Cardiology/American Heart Association Task Force on Clinical Practice Guidelines. Hypertension 71(6):1269-1324. https://doi.org/10.1161/hyp.0000000000 000066

29. Vassalotti JA, Stevens LA, Levey AS (2007) Testing for chronic kidney disease: a position statement from the National Kidney Foundation. Am J Kidney Dis 50(2):169-180. https://doi.org/10. 1053/j.ajkd.2007.06.013

30. Grundy SM, Brewer HB Jr, Cleeman JI, Smith SC Jr, Lenfant C (2004) Definition of metabolic syndrome: report of the National Heart, Lung, and Blood Institute/American Heart Association conference on scientific issues related to definition. Circulation 109(3):433-438. https://doi.org/10.1161/01.cir.0000111245. 75752.c6

31. Yeh HC, Lin YS, Kuo CC, Weidemann D, Weaver V, Fadrowski J, Neu A, Navas-Acien A (2015) Urine osmolality in the US population: implications for environmental biomonitoring. Environ Res 136:482-490. https://doi.org/10.1016/j.envres. 2014.09.009

32. Chang T, Ravi N, Plegue MA, Sonneville KR, Davis MM (2016) Inadequate hydration, BMI, and obesity among US adults: NHANES 2009-2012. Ann Fam Med 14(4):320-324. https://doi. org/10.1370/afm.1951

33. Perrier ET, Bottin JH, Vecchio M, Lemetais G (2017) Criterion values for urine-specific gravity and urine color representing adequate water intake in healthy adults. Eur J Clin Nutr 71(4):561563. https://doi.org/10.1038/ejcn.2016.269

34. Perrier ET, Buendia-Jimenez I, Vecchio M, Armstrong LE, Tack I, Klein A (2015) Twenty-four-hour urine osmolality as a physiological index of adequate water intake. Dis Markers 2015:231063. https://doi.org/10.1155/2015/231063

35. Enhorning S, Struck J, Wirfalt E, Hedblad B, Morgenthaler NG, Melander O (2011) Plasma copeptin, a unifying factor behind the metabolic syndrome. J Clin Endocrinol Metab 96(7):1065-1072. https://doi.org/10.1210/jc.2010-2981

36. Enhorning S, Wang TJ, Nilsson PM, Almgren P, Hedblad B, Berglund G, Struck J, Morgenthaler NG, Bergmann A, Lindholm E, Groop L, Lyssenko V, Orho-Melander M, Newton-Cheh C, Melander O (2010) Plasma copeptin and the risk of diabetes mellitus. Circulation 121(19):2102-2108. https://doi.org/10.1161/CIRCU LATIONAHA.109.909663

37. Roussel R, El Boustany R, Bouby N, Potier L, Fumeron F, Mohammedi K, Balkau B, Tichet J, Bankir L, Marre M, Velho G (2016) Plasma copeptin, AVP gene variants, and incidence of type 2 diabetes in a cohort from the community. J Clin Endocrinol Metab 101(6):2432-2439. https://doi.org/10.1210/jc.2016-1113

38. Brunkwall L, Ericson U, Nilsson PM, Enhörning S (2020) High water intake and low urine osmolality are associated with favorable metabolic profile at a population level: low vasopressin secretion as a possible explanation. Eur J Nutr. https://doi.org/10.1007/ s00394-020-02202-7

39. Armstrong LE, Soto JA, Hacker FT Jr, Casa DJ, Kavouras SA, Maresh CM (1998) Urinary indices during dehydration, exercise, and rehydration. Int J Sport Nutr 8(4):345-355. https://doi.org/10. 1123/ijsn.8.4.345

40. Waddell LS (2015) Chapter 187-Colloid osmotic pressure and osmolality monitoring. In: Silverstein DC, Hopper K (eds) Small animal critical care medicine (2nd edn). W.B. Saunders, St. Louis, pp 978-981. https://doi.org/10.1016/B978-1-4557-0306-7. 00187-2 
41. Guelinckx I, Vecchio M, Perrier ET, Lemetais G (2016) Fluid intake and vasopressin: connecting the dots. Ann Nutr Metab 68(Suppl 2):6-11. https://doi.org/10.1159/000446198

42. Johnson EC, Munoz CX, Le Bellego L, Klein A, Casa DJ, Maresh CM, Armstrong LE (2015) Markers of the hydration process during fluid volume modification in women with habitual high or low daily fluid intakes. Eur J Appl Physiol 115(5):1067-1074. https:// doi.org/10.1007/s00421-014-3088-2

43. McKenzie AL, Perrier ET, Guelinckx I, Kavouras SA, Aerni G, Lee EC, Volek JS, Maresh CM, Armstrong LE (2016) Relationships between hydration biomarkers and total fluid intake in pregnant and lactating women. Eur J Nutr. https://doi.org/10.1007/ s00394-016-1256-3

44. Johnson EC, Munoz CX, Jimenez L, Le Bellego L, Kupchak BR, Kraemer WJ, Casa DJ, Maresh CM, Armstrong LE (2016) Hormonal and thirst modulated maintenance of fluid balance in young women with different levels of habitual fluid consumption. Nutrients 8(5). https://doi.org/10.3390/nu8050302

45. Enhorning S, Tasevska I, Roussel R, Bouby N, Persson M, Burri P, Bankir L, Melander O (2017) Effects of hydration on plasma copeptin, glycemia and gluco-regulatory hormones: a water intervention in humans. Eur J Nutr. https://doi.org/10.1007/ s00394-017-1595-8

46. Lemetais G, Melander O, Vecchio M, Bottin JH, Enhorning S, Perrier ET (2017) Effect of increased water intake on plasma copeptin in healthy adults. Eur J Nutr. https://doi.org/10.1007/ s00394-017-1471-6

47. Enhörning S, Brunkwall L, Tasevska I, Ericson U, Persson Tholin J, Persson M, Lemetais G, Vanhaecke T, Dolci A, Perrier ET,
Melander O (2019) Water supplementation reduces copeptin and plasma glucose in adults with high copeptin: the $\mathrm{H}_{2} \mathrm{O}$ metabolism pilot study. J Clin Endocrinol Metab 104(6):1917-1925. https:// doi.org/10.1210/jc.2018-02195

48. Wannamethee SG, Welsh P, Papacosta O, Lennon L, Whincup PH, Sattar N (2015) Copeptin, insulin resistance, and risk of incident diabetes in older men. J Clin Endocrinol Metab 100(9):33323339. https://doi.org/10.1210/JC.2015-2362

49. Saleem U, Khaleghi M, Morgenthaler NG, Bergmann A, Struck J, Mosley TH Jr, Kullo IJ (2009) Plasma carboxy-terminal provasopressin (copeptin): a novel marker of insulin resistance and metabolic syndrome. J Clin Endocrinol Metab 94(7):2558-2564. https://doi.org/10.1210/jc.2008-2278

50. Enhörning S, Bankir L, Bouby N, Struck J, Hedblad B, Persson M, Morgenthaler NG, Nilsson PM, Melander O (2013) Copeptin, a marker of vasopressin, in abdominal obesity, diabetes and microalbuminuria: the prospective Malmö Diet and Cancer Study cardiovascular cohort. Int J Obes (Lond) 37(4):598-603. https:// doi.org/10.1038/ijo.2012.88

51. Enhorning S, Struck J, Wirfalt E, Hedblad B, Morgenthaler NG, Melander O (2011) Plasma copeptin, a unifying factor behind the metabolic syndrome. J Clin Endocrinol Metab 96(7):E1065-1072. https://doi.org/10.1210/jc.2010-2981

52. Abbasi A, Corpeleijn E, Meijer E, Postmus D, Gansevoort RT, Gans ROB, Struck J, Hillege HL, Stolk RP, Navis G, Bakker SJL (2012) Sex differences in the association between plasma copeptin and incident type 2 diabetes: the Prevention of Renal and Vascular Endstage Disease (PREVEND) study. Diabetologia 55(7):19631970. https://doi.org/10.1007/s00125-012-2545-X 